\title{
Using the $\mathrm{ACG}{ }^{\circledR}$ casemix system in population health management programs at Johns Hopkins L Dunbar* and M Sylvia
}

Address: Care Management, Johns Hopkins HealthCare, Glen Burnie, MD, USA

* Corresponding author

from 25th Patient Classification Systems International (PCSI) Working Conference

Fukuoka, Japan. II-I4 November 2009

Published: 5 November 2009

BMC Health Services Research 2009, 9(SuppI I):AI6 doi:I0.1186/1472-6963-9-SI-AI6

This abstract is available from: http://www.biomedcentral.com/I472-6963/9/SI/AI6

(c) 2009 Dunbar and Sylvia; licensee BioMed Central Ltd.

\section{Introduction}

Johns Hopkins HealthCare contracts with the US Department of Defense (DOD) to provide fully capitated healthcare services to 28,000 DOD beneficiaries. The health plan is known as the US Family Health Plan (USFHP). In order to understand and meet the healthcare needs of the USFHP population, Johns Hopkins HealthCare uses The ACG $^{\circledast}$ (Adjusted Clinical Group) Casemix System to perform a population health analysis. It then targets patients for a variety of population health-management interventions.

Programs such as Health Coaching, Disease Management and Case Management have been developed and implemented to help this population improve health, and reduce healthcare expenditures. The purpose of this presentation is to:

1) Present the results of the population analyses

2) Review the process for identifying and stratifying appropriate patients for interventions

3) Describe the management programs employed at Johns Hopkins HealthCare

4) Review the results of an intensive case-management program, Guided Care (GC), for patients with multimorbidity

\section{Methods}

Population analyses were performed annually to assess changes in population morbidity over time, and to create a population-management plan. Additionally, the ACG diagnostic and pharmacy predictive models were run monthly to identify and stratify individuals for a variety of population health-management programs. Health education and promotion, via books and web-based access to materials, were offered to patients with the lowest ACG risk scores.

Patients with moderate risk scores, or a single chronic disease, were offered health-coaching resources for lifestyle management to improve their health behaviors. Members with multiple chronic conditions, and high ACG risk scores, participated in GC, a nurse-led, patient-centered, comprehensive chronic-care program delivered in both the primary-care setting and the patient's home.

\section{Results}

Annual population analyses showed demographic and morbidity characteristics of the 28,000 patients. Mean ACG risk scores by primary care site, number of chronic conditions, and prevalence of chronic disease compared to national US benchmarks showed that morbidity is high in the US Family Health Plan population.

In 2006, we began a cluster-randomized, controlled trial of Guided Care in the mid-Atlantic region of the United States. This study was designed to measure the effects of GC on the quality of care for a multi-morbid population with high-risk scores on the outcomes of care for patients, 
families, primary-care practices, physicians, nurses, and healthcare insurers.

We hypothesized that 1) GC would improve patients' quality of care and physicians' satisfaction with care within 6 months, and 2) better quality of care would secondarily lead to improvements in patients' quality of life and efficiency of resource use - as well as to desirable outcomes for other stakeholders in chronic care.

Preliminary data indicated that Guided Care:

1) Improves the quality of patients' care. (After six months, GC patients were twice as likely as regularcare patients to rate the quality of their care highly. After 20 months, GC patients were more than twice as likely as regular-care patients to rate the quality of their care highly).

2) Reduces the use and cost of expensive services. (After the first eight months of the study, GC patients experienced, on average, 24\% fewer hospitals days, $37 \%$ fewer skilled nursing facility days, $15 \%$ fewer emergency department visits, and 29\% fewer home healthcare episodes. GC patients also experienced $9 \%$ more specialist visits; however, this is not considered statistically significant. Based on current Medicare payment rates, and GC costs, these differences in utilization produce net savings for healthcare payors.)

3) Reduces family caregiver strain. (After six months, the GC caregivers' "strain" and "depression" scores were lower than the comparison (regular care) caregivers' scores, especially among caregivers who provided more than 14 hours of weekly assistance.

4) Improves physicians' satisfaction with chronic care. (Compared to the physicians in the control group, the physicians who practiced GC for a year rated their satisfaction with patient/family communication, and their knowledge of their chronically-ill patients' clinical conditions, significantly higher.

\section{Conclusion}

The ACG ${ }^{\circledR}$ Casemix System was used effectively to perform population health analyses for the US Family Health Plan, a fully capitated 28,000 member health plan managed by Johns Hopkins HealthCare. The ACG Predictive Model identified and stratified the members into appropriate levels of population health-management intervention programs. As a program for members with multi-morbidity and the highest-risk scores, Guided Care improved patients' quality of care, physicians' satisfaction with care, patients' quality of life and efficiency of resource use. As well, it led to desirable outcomes for other stakeholders in chronic care.

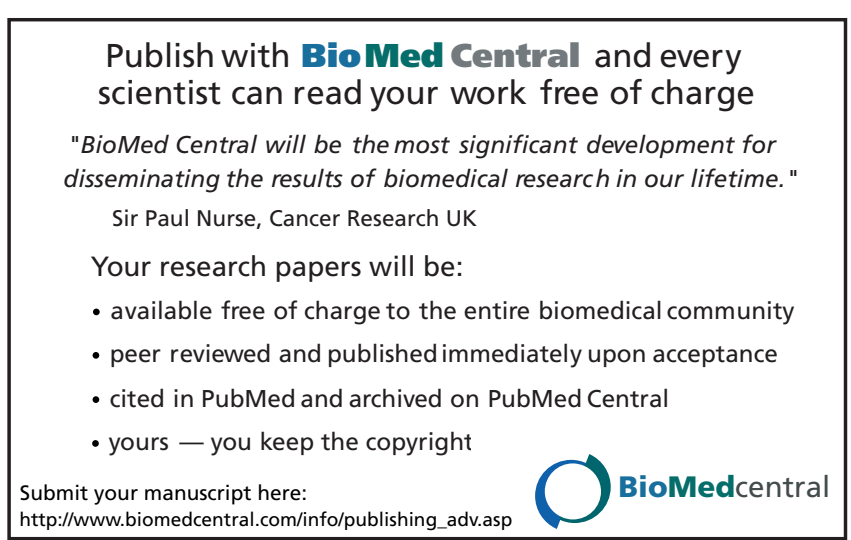

Page 2 of 2

(page number not for citation purposes) 